\title{
An loT-based prototype of a driverless bulldozer
}

\section{Carreras Guzman, Nelson Humberto; Mezovari, Adam Gergo; Yan, Ying}

Link to article, DOI:

10.1109/DCOSS.2019.00068

Publication date:

2019

Document Version

Peer reviewed version

Link back to DTU Orbit

Citation (APA):

Carreras Guzman, N. H., Mezovari, A. G., \& Yan, Y. (2019). An loT-based prototype of a driverless bulldozer. Paper presented at 2019 IEEE 15th International Conference on Distributed Computing in Sensor Systems, Santorini Island, Greece. https://doi.org/10.1109/DCOSS.2019.00068

\section{General rights}

Copyright and moral rights for the publications made accessible in the public portal are retained by the authors and/or other copyright owners and it is a condition of accessing publications that users recognise and abide by the legal requirements associated with these rights.

- Users may download and print one copy of any publication from the public portal for the purpose of private study or research.

- You may not further distribute the material or use it for any profit-making activity or commercial gain

- You may freely distribute the URL identifying the publication in the public portal

If you believe that this document breaches copyright please contact us providing details, and we will remove access to the work immediately and investigate your claim 


\section{An IoT-based prototype of a driverless bulldozer}

\author{
Nelson H. Carreras Guzman \\ Engineering Systems Group \\ Technical University of Denmark \\ Kgs. Lyngby, Denmark \\ nelca@dtu.dk
}

\author{
Adam Gergo Mezovari \\ Department of Electrical Engineering \\ Technical University of Denmark \\ Kgs. Lyngby, Denmark \\ s163169@student.dtu.dk
}

\author{
Ying Yan \\ Martin Nordal Petersen \\ Department of Photonics Engineering \\ Technical University of Denmark \\ Kgs. Lyngby, Denmark \\ yiya@fotonik.dtu.dk; \\ mnpe@fotonik.dtu.dk
}

\begin{abstract}
Recent developments in the field of Industry 4.0 have led to an increased interest in driverless mobile machinery. In industrial sectors such as agriculture, mining and construction, practitioners are demanding autonomous solutions to reduce operational costs and enhance efficiency and reliability. This paper demonstrates an implementation of Internet of Things (IoT) technologies to control a driverless mobile machinery prototype. After reviewing the technologies available for the IoT, we integrate a cloud application, communication protocols and a wireless communication network to control a small-scale bulldozer from a remote workstation. This solution is affordable, open-source, easyto-install and scalable, providing a platform to implement in several industrial applications.
\end{abstract}

Keywords-Internet of Things (IoT), mobile machinery, Industry 4.0, cloud computing, communication protocol

\section{INTRODUCTION}

Rapid developments in wireless communication technologies, embedded systems and Internet usage have enabled the deployment of Internet of Things (IoT) solutions to monitor critical infrastructures and industrial activities [1]. As a result, the notions of cities, transportation and agriculture as 'smart' systems are becoming a reality. For example, wireless sensor network (WSN) integrated with IoT technologies are allowing continuous monitoring of the physical environment for water management, smart grids, transportation networks, croplands, among others [2], [3].

In parallel, cyber-physical systems (CPS) are making possible the control of physical and engineered systems, integrating physical world applications with real-time communications and computational processes [4], [5]. The developments in the fields of IoT and CPS are key factors leading to a forth Industrial Revolution, also referred as Industry 4.0 [6], [7]. In smart vehicles, their applications include autonomous cars, buses and trains for urban mobility; trucks, vessels and drones for logistics; and "mobile machinery" [8] such as tractors and heavy-duty vehicles in the agriculture, mining and construction industries.
Some main differences between driverless mobile machinery with respect to autonomous cars include the addition of complex field operations and remote monitoring and control. Nevertheless, driverless mobile machinery mainly operate in controlled environments owned by the stakeholders, allowing simpler configurations for fleet coordination with respect to autonomous vehicles in public road networks [9], [10]. Overall, these characteristics make mobile machinery cases attractive for automation, being proved already as economically feasible in some applications [11], [12]. The current high costs in precise positioning systems (e.g. RTK GPS) and laser vision sensors (e.g. Lidar) are progressively reduced due to competitive innovation [13].

Assessing the technological developments in mobile machinery, Wilson [14] studied 50 years of guidance systems for agricultural vehicles, concluding that the integration of global positioning system and detection sensors in the vehicle was the best solution to replace human drivers. More recently, Mousazadeh [15] reviewed six classes of navigation systems for agricultural machinery, while highlighting important concerns such as safety, economic feasibility and standardization. In experimental deployments, Stentz et al. [12] illustrated a system for automation and tested a semi-autonomous tractor equipped with GPS and sensors to detect obstacles and stop to avoid collision.

Nowadays, the technological trends privilege remote control and fully autonomous operations. Nørremark et al. [16] developed an autonomous control system to perform hoeing within crop rows without collision with plants. Moreover, companies such as Caterpillar in the mining sector [17] and $\mathrm{CNH}$ Industrial in the agricultural sector [18] currently provide solutions in the market for semi- and fully-autonomous mobile machinery applications. However, none of these reviewed applications integrates IoT-based solutions to monitor and control these driverless vehicles.

Overall, from this review we assess that researchers have mainly focused on industrial IoT-based applications for information awareness through monitoring. Nevertheless, these technologies are also suitable for integration in the automation 
of mobile machineries, providing a platform to monitor and control remotely in real-time.

Thus, this paper demonstrates an implementation of IoT technologies to control a small-scale driverless bulldozer. We integrate a cloud application, communication protocols and a wireless communication network to a control system in the vehicle, enabling remote controlling of semi-autonomous mobile machineries from an affordable, open-source, easy-toinstall and scalable platform. While focusing on the integration of the different communication technologies and the cloud application, we also demonstrated the physical implementation and operation in a small-scale driverless bulldozer. The bulldozer is controlled remotely assigning road segments to drive in a work field. Furthermore, the vehicle is equipped with a safety stop function when encountering an obstacle in front of it, allowing two-way communications between the controller in the vehicle and a user interface (UI) designed ad hoc in a cloud application.

This paper is organised as follows. Section 2 describes the communication technologies surveyed, providing the reasoning to select the implemented ones. Section 3 presents the physical prototype, the conceptual control design and the developed user interface. Section 4 illustrates the results and the challenges encountered during the process. Section 5 suggests further developments for scaling the project, and finally Section 6 concludes.

\section{DesCriPTION OF COMMUNICATION TECHNOLOGIES}

To realize the remote operation of the developed prototype, we needed to establish a wireless communication with the cloud application. Because this kind of vehicles require reliable monitoring, the connection needed to be not only persistent, but also capable of sending sufficient amount of data with a high frequency. The applied communication technology also needed to cover wide areas to avoid limitations in the size of the work fields. The different communication technologies considered are compared in Table I, adapted from [19], [20], and [21].

TABLE I. COMPARISON OF NETWORK TECHNOLOGIES

\begin{tabular}{|l|l|l|l|l|l|}
\hline Feature & $\begin{array}{l}\text { Cellular } \\
\text { (GPRS) }\end{array}$ & $\begin{array}{l}\text { Wifi } \\
\mathbf{( 8 0 2 . 1 1 a h )}\end{array}$ & LoRa & Sigfox & NB-IoT \\
\hline $\begin{array}{l}\text { Max } \\
\text { range }\end{array}$ & $15 \mathrm{~km}$ & $1 \mathrm{~km}$ & $15 \mathrm{~km}$ & $50 \mathrm{~km}$ & $15 \mathrm{~km}$ \\
\hline $\begin{array}{l}\text { Max data } \\
\text { rate } \\
\text { (up/down) }\end{array}$ & $\begin{array}{l}20 / 114 \\
\mathrm{kbps}\end{array}$ & $\begin{array}{l}7.8 / 7.8 \\
\text { Mbps }\end{array}$ & $\begin{array}{l}100 / 100 \\
\mathrm{kbps}\end{array}$ & $\begin{array}{l}100 / 100 \\
\mathrm{kbps}\end{array}$ & $\begin{array}{l}64 / 128 \\
\mathrm{kbps}\end{array}$ \\
\hline Scalability & High & Limited & $\begin{array}{l}\text { Very } \\
\text { Low }\end{array}$ & Low & High \\
\hline $\begin{array}{l}\text { Power } \\
\text { need }\end{array}$ & High & High & Low & Low & Medium \\
\hline Cost & Low & Medium & High & Low & Medium \\
\hline $\begin{array}{l}\text { Frequency } \\
\text { band }\end{array}$ & Reserved & Open & Open & Open & Reserved \\
\hline
\end{tabular}

\section{A. GPRS}

Considering the above comparison table, we selected the General Packet Radio Service (GPRS) technology, an extension of the Global System for Mobile (GSM) technology with support for data features, to connect the internet via the internet protocol suite (TCP/IP). Cellular networks provide suitable field coverage for moving vehicles. The cost of the implementation is also beneficial. With an inexpensive GSM module and a SIM card, the client is authorised to use the wellestablished cellular networks of telecommunication companies. Considering data rate and scalability, cellular networks proved to be a preferable choice. Moreover, a frequency band is reserved for this technology, making it less prone to jamming and unauthorised access. Operators manage and maintain the infrastructure, providing a reliable and secure connection.

The power consumption of a cellular module is significantly high comparing to other IoT communication technologies. However, in a real application, a diesel engine would be running the bulldozer. Therefore, power need is not a significant issue in this application. In our prototype, a SIM800L GSM module was attached to the microcontroller as a modem to connect the vehicle to the cellular network. To control the GSM modem, we used standard attention (AT) commands.

\section{B. MQTT}

The open-source Message Queuing Telemetry Transport (MQTT) is a machine-to-machine connectivity protocol. It is based on a publish-subscribe model, providing two-way communication through TCP/IP. It was originally developed by IBM for low bandwidth, resource-constrained devices in embedded systems. Because of its low complexity and low power draw, it is a suitable choice for IoT applications. It offers a compact binary packet payload, which is much simpler than other messaging technologies such as HTTP. For this project, we chose the MQTT protocol because it provides a convenient integration with several cloud applications. Nevertheless, other IoT protocols are also available (see [22]).

\section{SYSTEM SETUP AND DIAGRAM}

\section{A. Bulldozer physical built system}

The bulldozer is an original design based on the Detecting Robot form proposed by the Makeblock Ultimate 2.0 kit. Additionally, a blade mechanism was included to provide the blade functionality of a bulldozer. For this reason, the assembly had to be tailored to provide physical space for the blade mechanism, while allowing for a clear space for the ultrasonic sensor to detect obstacles in front of the bulldozer. In Fig.1, the main components of the prototype are illustrated.

A set of beams and plates joined by screws compose the chassis. As mechanical moving parts, a configuration of gears and tires transmit the torque from the motors to displace the bulldozer on the ground. Likewise, a gear transmits the rotations from a motor to the blade mechanism to move it up and down. 

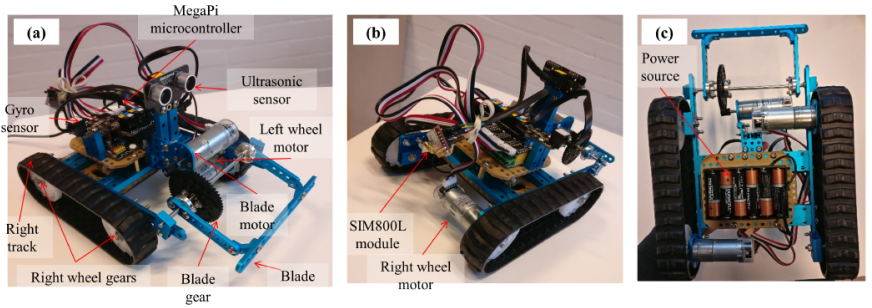

Fig. 1. Prototype main views and parts explained (a) Front view; (b) Back view; (c) Bottom view

To avoid latency issues in the critical control functions, the control logic was allocated in the microcontroller directly on the bulldozer. An ultrasonic sensor was allocated facing front to detect obstacles ahead when the bulldozer is moving forward. Furthermore, a gyro sensor provides the current heading from its yaw measurement, used to calculate the initial turn. In total, three encoder motors were installed; two of them to move tire gears (one at each side), and one motor to move the blade mechanism. The sensors and the GSM module were connected using serial communications, while the motors were connected through motor drivers.

\section{B. Layered functional diagram}

The overall system can be represented using a layered framework for cyber-physical systems (CPS) adapted from [23]. This framework subdivides the system in three layers: cyber, cyber-physical, and physical. On the one hand, the physical layer is composed by parts and processes that are not materialized by computers or digital networks. Instead, they are accomplished by energy transformations between mechanical parts, as well as chemical or biological processes. On the other hand, the cyber layer refers to the computations, communications, and supervisory control processes that are not directly in contact with the physical processes. As an intermediate level, the cyber-physical layer is composed by reactive control functions performed by the embedded system, as well as sensors and actuators as inputs and outputs from the control loop. Finally, the CPS as a whole interacts with the cyber and physical environments.

Using this multi-layered framework, Fig. 2 illustrates the driverless bulldozer system and environments. In the cyber layer, the human operator inserts the routes to be followed by the bulldozer through the dedicated UI. The programmer is also depicted, considering his interaction with the UI and the microcontroller as developer. The information inserted by the operator is transmitted using MQTT via GPRS communications to the microcontroller. Then, the algorithms embedded in the controller process this information, executing the route in the physical layer using sensors as inputs and actuators as outputs. When completing the route segment, or if the system encounters an obstacle, the bulldozer stops and sends its position and a message to the UI.

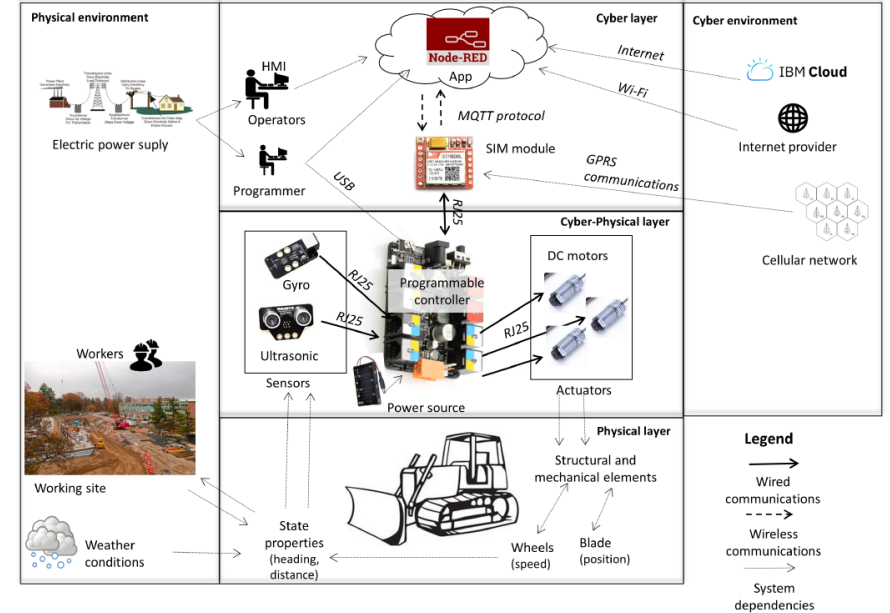

Fig. 2. System representation as multi-layered CPS and environments

\section{Cloud application and user interface}

As MQTT broker, the open-source message broker Mosquitto [24] was configured on the microcontroller. Seven different topics were introduced to realize the two-way communication and separate the different information flows. Because the JavaScript Object Notation (JSON) is a popular and well-structured format, all payloads are sent in this format.

To design the UI, we used the Node-RED programming tool integrated with IBM Cloud. As shown in the UI in Fig. 3, the operator finds the current status and position of the vehicle in the header of the UI. The status has three states: Idle, Running and Stop. Next to the position widget, three buttons (STOP, RESUME and RESET) are placed to assure on-demand access to basic control functions.

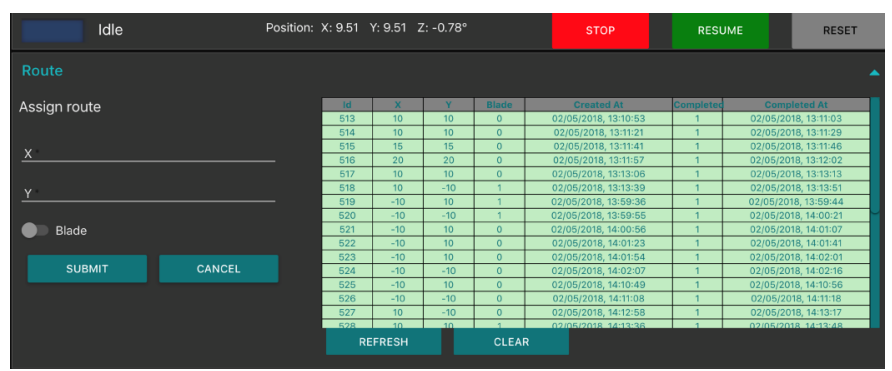

Fig. 3. Developed user interface in cloud platform

The next part of the UI refers to the route segments. First, there is a simple form where the operator can assign routes to the bulldozer by defining the target $\mathrm{X}$ and $\mathrm{Y}$ coordinates together with a blade position. Next to the form, there is a table listing all the previous routes assigned. In the table, completed route segments are marked as green and have the completed flag set. The coded flow diagram for route assignment is illustrated in Fig.4. 


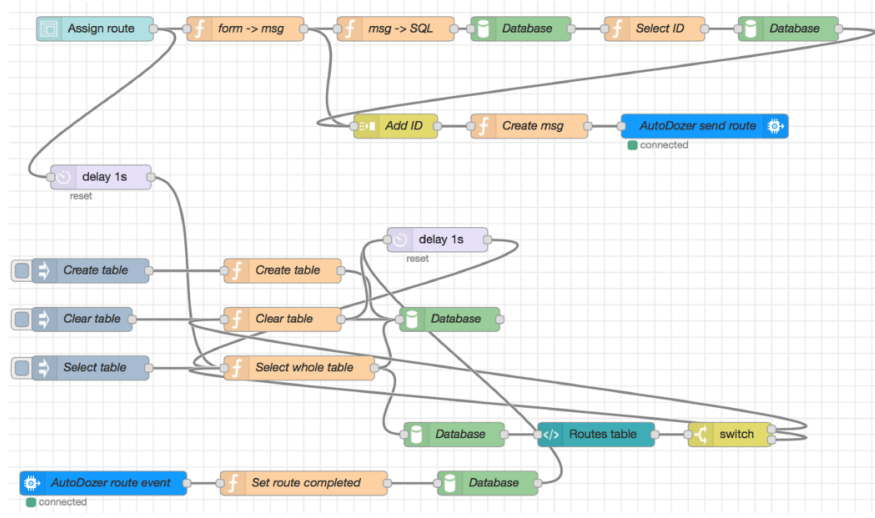

Fig. 4. Flow diagram for route assignment in cloud platform

The last two elements of the user interface are two tables, which are not visible in the figures. The first one is listing the positions of detected obstacles, while the second one is a history of the bulldozer's position.

\section{Conceptual design of physical processes}

Beyond the integration of the different communication technologies and the cloud application, we also demonstrated the physical implementation and operation of the system in the small-scale driverless bulldozer. As a conceptual design choice, the route handling was conceived as four sequential control function modes.

\section{1) Route assignment}

The operator inserts the route assignment through the dedicated UI in Node-RED. These route assignments are a sequence of linear segments for the bulldozer to drive forward and the position of the blade during each segment. The routes inserted by the operator are transmitted to the microcontroller using MQTT via GPRS communications. Subsequently, the algorithms embedded in the controller process this information as inputs for the control functions.

\section{2) Turn while detecting obstacle}

To perform the route assignment, the first function is to turn to align its front side to the direction where it will to drive forward. In terms of the control algorithm, the bulldozer is conceived in a Cartesian plane with current position and current heading. Therefore, the controller has to compare its current position to the target position established in the route assignment and decide on the direction and amount of degrees to turn. If sensor readings indicate that no obstacles are impeding a safe manoeuvre, the controller sends commands to the actuators controlling the wheels to turn. If an obstacle is detected, the bulldozer stops and sends an alert to the UI informing its decision to stop for safety reasons. When the obstacle is no longer present, the operator can resume the current route assignment and the bulldozer with proceed with the execution.

\section{3) Blade to position}

When completing the turn, the bulldozer's front side is facing the direction to drive forward. If the route assignment includes a new position of the blade, the controller sends a command to the blade mechanism and put the blade in the assigned position.

\section{4) Drive forward while detecting obstacle}

Finally, the controller activates the motors in the wheels and drives the bulldozer forward until the destination point. Again, if the sensor detects an obstacle during the route, the bulldozer stops and sends its position and a message to the UI. When finishing the current route, the process restarts for the following route assignment. During the whole process, the controller continuously sends its position and status to the UI and reports it in the respective tables for monitoring purposes.

In Fig. 5, we represent the flowchart for the "drive forward while detecting obstacle" mode algorithm.

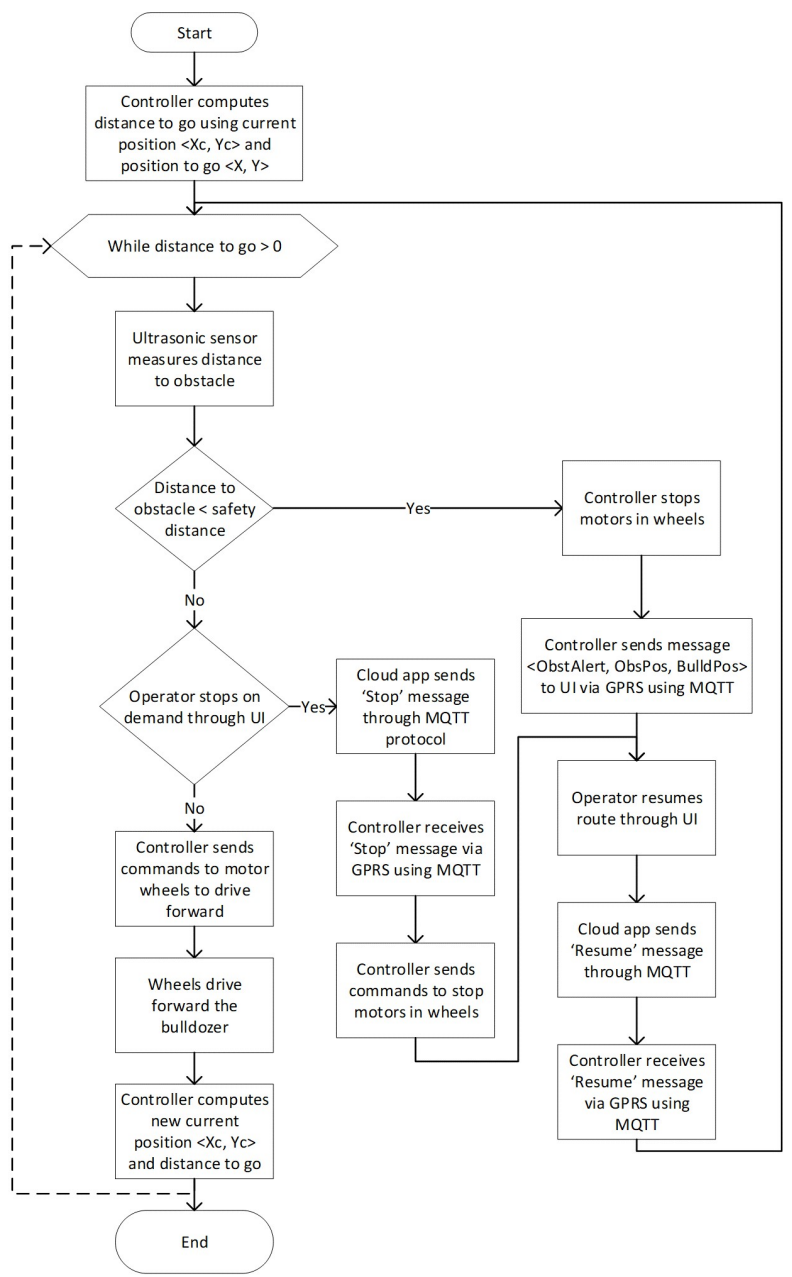

Fig. 5. Conceptual design of control function mode "Drive forward while detecting obstacle".

\section{RESULTS AND CHALLENGES}

Two-way communications between the cloud application and the microcontroller were successfully deployed through the GPRS network using the MQTT protocol. Furthermore, we successfully deployed a dedicated UI in Node-RED for route assignment and on demand command functions. A database of assigned routes (completed, or to be completed), obstacles position and bulldozer position was automatically filled with 
systematic inputs sent from the microcontroller. Communication between the control unit and sensors, actuators and modules were successfully established using serial communication. Despite the use of a cellular network instead of a Low Power Wide-Area Network (LPWAN), the use of a cloud application and the MQTT messaging protocol to manage sensors and actuators through a microcontroller provides a strong argument to categorize this project as an IoT application.

We encountered some issues to establish the serial communication between the microcontroller and the GSM module. Nevertheless, these issues were mainly associated to the limitations of prototype application. The SIM800L is a very sensitive and power-consuming module, requiring a proper power supply to keep alive a robust GPRS connection. The module needs $3.4-4.4 \mathrm{~V}$ and demands up to $2 \mathrm{~A}$ when registering to the network. We managed to provide these conditions through the I/O pins of the microcontroller. Later on, the MQTT connection was not entirely reliable resulting in connection and message losses. Despite discrete disconnections, the communications worked most of the time.

While focusing on the integration of the different communication technologies and the cloud application, we also demonstrated the physical implementation in a small-scale driverless bulldozer. We managed to operate the system successfully in a controlled environment, driving route segments and avoiding collisions with obstacles.

\section{FURTHER DEVELOPMENTS AND IMPROVEMENTS}

In this implementation, we were not aiming at designing a prototype with the complex control capabilities of an autonomous vehicle. Instead, we demonstrated that the IoT technologies provided the necessary conditions for the bulldozer to operate in the physical world according to the communication and control commands provided by the operator using the UI. In the following paragraphs, however, we describe potential improvements to the prototype versions that could be explored in further work for more realistic applications.

For this prototype, a set of open-loop controls were developed to execute the route segments. This decision to use open-loop controls instead of closed-loop controls was taken considering the limitations in execution accuracy and fluidity. In fact, a closed-loop control was designed as a first turn logic, with dynamic measurements and heading corrections throughout the route execution. However, this solution proved inconvenient in small turns performed in controlled steps, considering that the low voltage triggered by the motors to perform small rotations did not provide enough torque to overcome static friction (i.e. stiction) between the tires and the ground surface. This design choice is not fit to be scaled to a real-scale system for poor efficacy and safety-related considerations. Conversely, we suggest closed-loop controls with feedback correction in each phase of the process.

To deliver a more reliable route execution, the gyro sensor is not recommended as a navigation device, considering the use of relative measurements and the need for recurrent calibrations. Instead, a solid-state compass would provide absolute readings at all times, which are not dependent on the calibration at the initial state. Moreover, real-time kinematic (RTK) positioning would give accurate GPS coordinate readings to assess the vehicle position in a global reference system, enabling the necessary inputs for feedback control during route execution. Geo-fencing could also be implemented to avoid accidental or deliberate manipulations outside the working site.

Finally, this application could be extended for a fleet of mobile machineries. This would require additional coordination logic to avoid accidents and implement cooperative functions among the different machineries. Moreover, considering that mobile machinery fleets in real-scale would be safety-critical applications, the need for redundancy in communication and control platforms is recommended. Security measures should also be included in further work, especially to protect the system from attackers motivated to manipulate the system and induce physical harm scenarios.

\section{CONCLUSIONS}

This paper investigated a development of a low-cost, scalable, driverless bulldozer prototype using open-source IoT protocols and cloud computing. In this research, we tested the current inexpensive technologies to operate semi-autonomous mobile machineries in the context of Industry 4.0. After surveying the features of the available communication technologies and cloud platforms, we were able to implement successfully the control and monitoring system in a bulldozer prototype. Despite the limitations encountered due to the smallscale application, we discussed alternative solutions to overcome them in real-scale cases; namely, to provide dependable, safe and secure execution through more suitable control devices and redundancy in communication and control capabilities. This study will serve as a base for future studies of autonomous mobile machineries, enabling an extension of the proposed configuration to semi-autonomous fleets in construction, agricultural, and mining contexts, among others.

\section{REFERENCES}

[1] X. Ge, F. Yang, and Q. L. Han, "Distributed networked control systems: A brief overview," Inf. Sci. (Ny)., vol. 380, pp. 117-131, 2017.

[2] L. Atzori, A. Iera, and G. Morabito, "The Internet of Things: A survey," Comput. Networks, vol. 54, pp. 2787-2805, 2010.

[3] J. Gubbi, R. Buyya, S. Marusic, and M. Palaniswami, "Internet of Things (IoT): A vision, architectural elements, and future directions," Futur. Gener. Comput. Syst., vol. 29, no. 7, pp. 1645-1660, 2013.

[4] R. Rajkumar, I. L. I. Lee, L. S. L. Sha, and J. Stankovic, "Cyber-physical systems: The next computing revolution," Des. Autom. Conf. (DAC), 2010 47th ACM/IEEE, pp. 0-5, 2010.

[5] E. A. Lee and S. A. Seshia, Introduction to Embedded Systems: A CyberPhysical Systems Approach, Second Edi. The MIT Press, 2017.

[6] L. Monostori, "Cyber-physical production systems: Roots, expectations and R\&D challenges," Procedia CIRP, vol. 17, pp. 9-13, 2014.

[7] N. Jazdi, "Cyber physical systems in the context of Industry 4.0," Proc. 2014 IEEE Int. Conf. Autom. Qual. Testing, Robot. AQTR 2014, pp. 1-4, 
2014.

[8] European Commission, "Mobile machinery," Growth: Internal Market, Industry, Entrepreneurship and SMEs, 2018. [Online]. Available: https://ec.europa.eu/growth/sectors/mechanicalengineering/mob_machinery_en. [Accessed: 05-Jul-2018].

[9] T. Simonite, "Mining 24 Hours a Day with Robots," MIT Technology Review, 2016.2 [Online]. Available: https://www.technologyreview.com/s/603170/mining-24-hours-a-daywith-robots/. [Accessed: 06-Jul-2018].

[10] C. Underwood, "The Future of AI in Heavy Industry - Agriculture, Construction, Mining, and Beyond," Techemergence, 2018. [Online]. Available: https://www.techemergence.com/future-ai-in-heavy-industry/. [Accessed: 06-Jul-2018].

[11] S. M. Pedersen, S. Fountas, H. Have, and B. S. Blackmore, "Agricultural robots - System analysis and economic feasibility," Precis. Agric., vol. 7, no. 4, pp. 295-308, 2006.

[12] A. Stentz, C. Dima, C. Wellington, H. Herman, and D. Stager, "A System for Semi - Autonomous Tractor Operations," Auton. Robots, vol. 13, pp. $87-104,2002$

[13] D. Muoio, "Google just made a big move to bring down the cost of selfdriving cars," Business Insider, 2017. [Online]. Available: http://www.businessinsider.com/googles-waymo-reduces-lidar-cost-90in-effort-to-scale-self-driving-cars-2017-1 $\mathrm{r}=\mathrm{US} \& \mathrm{IR}=\mathrm{T} \& \mathrm{IR}=\mathrm{T}$. [Accessed: 06-Jul-2018].

[14] J. N. Wilson, "Guidance of agricultural vehicles - A historical perspective," Comput. Electron. Agric., vol. 25, no. 1-2, pp. 3-9, 2000.

[15] H. Mousazadeh, "A technical review on navigation systems of agricultural autonomous off-road vehicles," J. Terramechanics, vol. 50, no. 3, pp. 211$232,2013$.

[16] M. Nørremark, H. W. Griepentrog, J. Nielsen, and H. T. Søgaard, "The development and assessment of the accuracy of an autonomous GPS-based system for intra-row mechanical weed control in row crops," Biosyst. Eng., vol. 101, no. 4, pp. 396-410, 2008.

[17] Caterpillar Inc., "Caterpillar Mining Technology: Autonomous Trucks," 2014. [Online]. Available: https://www.youtube.com/watch?v=VkanknGePhc. [Accessed: 26-Apr2018].

[18] CNH Industrial, "The CNH Industrial Autonomous Tractor Concept (Full Version)," $2016 . \quad$ [Online]. Available: https://www.youtube.com/watch?v=T7Os5Okf3OQ. [Accessed: 26-Apr2018].

[19] I. Tardy, N. Aakvaag, B. Myhre, and R. Bahr, "Comparison of wireless techniques applied to environmental sensor monitoring," 2017. [Online]. Available:

https://brage.bibsys.no/xmlui/handle/11250/2436270?show=full.

[20] B. Vejlgaard, M. Lauridsen, H. Nguyen, I. Z. Kovacs, P. Mogensen, and M. Sorensen, "Coverage and Capacity Analysis of Sigfox, LoRa, GPRS, and NB-IoT," IEEE Veh. Technol. Conf., vol. 2017-June, 2017.

[21] H. Wang and A. O. Fapojuwo, "A Survey of Enabling Technologies of Low Power and Long Range Machine-to-Machine Communications," IEEE Commun. Surv. Tutorials, vol. 19, no. 4, pp. 2621-2639, 2017.

[22] A. Al-Fuqaha and M. Guizani, "Internet of things: A survey on enabling technologies, protocols, and applications," IEEE Commun. Surv. Tutorials, vol. 17, no. 4, pp. 2347-2376, 2015.

[23] A. Humayed, J. Lin, F. Li, and B. Luo, "Cyber-Physical Systems Security - A Survey,” IEEE Internet Things J., vol. 4, no. 6, pp. 1802-1831, 2017.

[24] Eclipse Foundation, "Eclipse Mosquitto" ${ }^{\mathrm{TM}}$. An open source MQTT broker." [Online]. Available: https://mosquitto.org/. [Accessed: 01-Aug2018]. 